\section{Épissothérapie et thalassémies}

Dominique Labie, Jean-Claude Kaplan
Inserm U567-UMR CNRS 8104, Institut Cochin, 24, rue du Faubourg Saint-Jacques, 75014 Paris, France. dominique.labie@inserm.fr jean-claude.kaplan@inserm.fr
> La manipulation thérapeutique de l'épissage permet de rétablir au niveau de I'ARN messager mûr un cadre de lecture interrompu par un défaut génomique quelconque. Ce rétablissement peut être notamment obtenu par saut d'exon (exon skipping), ou par neutralisation d'un site d'épissage aberrant. Dans les deux cas les effecteurs utilisés sont des oligonucléotides antisens (AON pour antisense oligonucléotides) complémentaires des sites à neutraliser. Cette stratégie de reprogrammation des ARN prémessagers, proposée dès 1993 par Riszard Kole (Chapel Hill) [1], n'a progressé que lentement au fil des années. Pendant plus de dix ans les preuves expérimentales de ces concepts in vitro et ex vivo se sont accumulées [2], sans être concrétisées par des essais cliniques. On assiste depuis quelques années à une accélération des progrès, essentiellement liées à : (1) une optimisation de la chimie des AON et de leur adressage $[2,3]$; (2) une rationalisation du choix des cibles [4]; (3) un approfondissement des connaissances sur l'épissage normal et pathologique [5]. En raison de la structure modulaire éminemment favorable du gène $D M D$ (Duchenne muscular dystrophy) et de la dystrophine, c'est l'«épissothérapie » par saut d'exon des dystrophinopathies (myopathies de Duchenne/Becker) qui est la plus avancée expérimentalement in vivo $[6,7]$ et qui a donné lieu aux premiers essais cliniques $[8,9]$.

A priori l'épissothérapie des bêta-thalassémies ne relève pas du saut d'exon, car ni la structure du gène de la bêtaglobine $(H B B)$ ni sa pathologie ne s'y prêtent. Cependant, il est des variants où la rupture du cadre de lecture résulte de mutations créant des néosites d'épissage $[10]^{1}$. Ici le retour à un patron d'épissage normal pourrait être obtenu en masquant ces sites par des AON ciblés. Plusieurs articles récents font état de preuves de concept enfin obtenues in vivo dans un modèle murin $[11,12]$ et laissent espérer une possible application en clinique.

\section{Les bêta-thalassémies}

Ce sont sans doute les maladies génétiques les plus fréquentes au monde. Elles résultent de mutations très diverses, et au moins 200 mutations différentes ont été décrites [13], entraînant un déficit partiel ou total de synthèse des chaînes de bêta-globine dont les conséquences cliniques sont de gravité variable [10]. L'abord thérapeutique est le plus souvent un régime transfusionnel à vie, combiné à des chélateurs du fer. La greffe de moelle osseuse, seul traitement correcteur, est d'usage limité (coût, rareté des donneurs, risques inhérents). Les espoirs fondés sur la réactivation du gène $H B F$ codant l'hémoglobine fœtale ont été déçus [10] et la thérapie génique «classique» des hémoglobinopathies n'a pas encore fait ses preuves en clinique [14]. En matière d'épissothérapie, nous avons vu que seules peuvent être retenues celles des bêta-thalassémies qui résultent de l'activation mutationnelle d'un site cryptique d'épissage (Tableaul). Si le nombre des mutations de ce type est limité, leur incidence épidémiologique n'est pas négligeable, car elles

${ }^{1}$ II faut les distinguer des mutations exoniques ou juxta-exoniques affectant les sites consensus d'épissage (donneurs, accepteurs, activateurs, branchement) qui ne sont pas encore accessibles à l'épissothérapie dont il est question ici. concernent au moins $10 \%$ des patients atteints d'une pathologie de l'hémoglobine. Parmi les mutations candidates à une épissothérapie, celles qui siègent dans l'IVS2 (intervening sequence 2) représentent un modèle particulièrement intéressant et c'est sur lui que se sont concentrés les efforts. En effet, les mutations aux nucléotides 654, 705 et 745 sont toutes trois créatrices d'un site donneur, et activent toutes trois un même site cryptique accepteur situé au nucléotide 579 (Figure 1). Le résultat est l'inclusion dans le préARNm d'un pseudo-exon de taille variable qui, dans les trois cas, interrompt le cadre de lecture. La mutation IVS2-654 a été le modèle le plus étudié, à cause de sa très grande fréquence en Chine et en Asie du Sud-Est [11]. Si elle n'engendre pas un phénotype nul $\left(\beta^{0}\right.$-thal), c'est parce qu'il existe un phénomène d'échappement avec utilisation très minoritaire des sites normaux [15]. La première tentative de correction du transcrit primaire à l'aide d'un AON spécifique remonte à 1993 [1]. Les auteurs avaient obtenu la restauration d'un épissage correct en ciblant en système acellulaire, soit le site 5' nouvellement créé, soit le site 3' cryptique activé. Dans les années suivantes, le bien-fondé de cette approche a été vérifié expérimentalement dans des systèmes cellulaires: cellules HeLa, puis précurseurs érythroïdes de sujets thalassémiques [16-19]. Le passage à l'expérimentation animale in vivo a été franchi grâce à des souris transgéniques dont un des deux locus bêta globine avait été remplacé par le locus bêta humain portant la mutation IVS2-654 [20]. Ces animaux sont hétérozygotes 


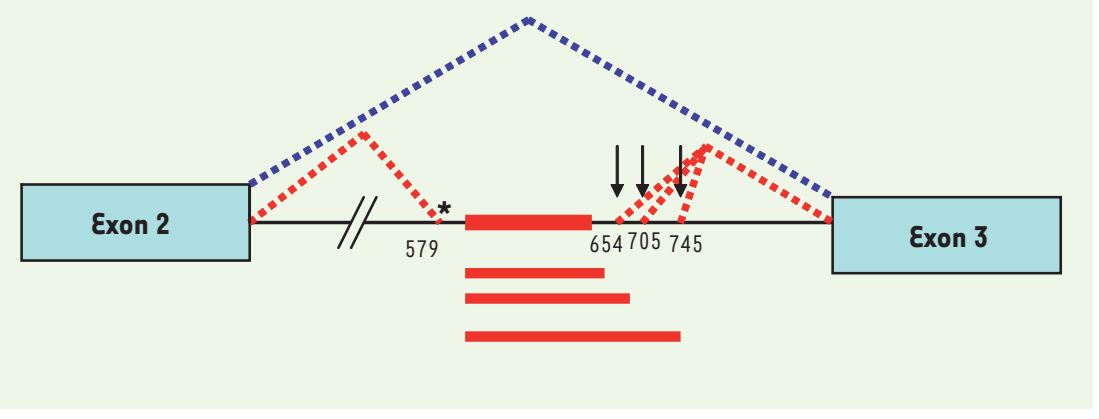

pour la mutation dans un contexte humain (l'homozygotie serait létale). Bien qu'hétérozygotes, ces souris dites $H b b^{\text {th- }-4} / \mathrm{Hbb}^{+}$présentent un phénotype thalassémique. Elles n'expriment pas d'HbA humaine. Après une série d'expériences effectuées ex vivo [17], une équipe de Shanghai a enfin apporté la preuve d'un effet bénéfique clinique et biologique de l'épissothérapie chez l'animal entier [11]. Ce résultat a été obtenu chez des souris $\mathrm{Hbb}^{\text {th- } 4} / \mathrm{Hbb}^{+}$ [20] ayant intégré, par transgenèse dans des ovocytes fécondés, un vecteur lentiviral à double effet, portant à la fois une séquence codant I'AON spécifique de la mutation humaine IVS2654 , et une séquence codant un shARN (sh : short hairpin) destiné à diminuer l'excès d'ARNm de la chaîne alpha globine. Même si cette ingénieuse prouesse expérimentale apportait la première preuve de concept d'un effet thérapeutique in vivo, on était encore loin d'une stratégie applicable en clinique. Le travail récent de l'équipe de Chapel Hill [12] franchit un pas décisif puisqu'il obtient un effet clinique avec synthèse d'HbA par un traitement de saut d'exon. Des souris $\mathrm{Hbb}^{\text {th-4 }} / \mathrm{Hbb}^{+}$[20] ont reçu pendant 3 semaines ( 4 jours consécutifs, 3 jours d'arrêt) par injection intraveineuse $(25 \mathrm{mg} / \mathrm{kg})$ un oligonucléotide antisens long de $18 \mathrm{nt}$ dirigé contre la mutation IVS2-654 (Figure 1). L’AON utilisé est un dérivé phosphorodiamidate morpholino conjugué à un peptide chargé positivement («SS0 654-P005 » dont la composition n'est pas révélée) destiné à favoriser la pénétration nucléaire. Par comparaison spécifique on a constaté la restauration de l'épissage normal $(x 6)$ et sa traduction en $\mathrm{HbA}$ (environ $10 \%$ ), ainsi que la présence d'hémoglobine chimère $\operatorname{souris}(m)$-homme $(h)\left(m \alpha^{2} h \beta^{2}\right)$. On observait en même temps une remontée du taux global d'hémoglobine et une amélioration morphologique des globules rouges. L'interprétation quantitative doit tenir compte de deux facteurs: (1) les souris étant hétérozygotes, seul un allèle a été corrigé ; (2) la durée de vie des globules rouges murins étant d'environ 100 jours, un traitement de trois semaines n'a pu corriger que moins du tiers des cellules. La valeur observée de $10 \%$ d'HbA est donc proche de la valeur théorique. Aucun signe de toxicité hépatique ou rénale n'a été constaté, ni des signes de stimulation immunitaire (absence d'anticorps spécifiques, pas de stimulation des lymphocytes).

\section{Perspectives}

\section{Les autres mutations responsables de thalassémies}

On pourrait envisager d'élargir le domaine d'application aux thalassémies fréquentes (Tableaul) comme celles causées par la mutation IVS1-110, très répandue dans le bassin méditerranéen oriental (Grèce, Chypre, Liban, Turquie, Égypte) [10]. C'est une thalassémie sévère dont la prise en charge thérapeutique est médicalement et éconoavec les témoins traités par un $A O N$ non
Figure 1. Schéma de l'épissage au niveau du deuxième intron de la $\boldsymbol{\beta}$-globine. L'épissage normal est figuré en pointillé bleu. Les épissages aberrants sont figurés en pointillé rouge. Les flèches verticales indiquent le site des mutations qui toutes trois créent un site donneur. L'étoile indique le site accepteur cryptique, le même activé par les trois mutations. Le résultat de ces épissages aberrants est l'inclusion d'un pseudo-exon, de taille variable selon la mutation (trait rouge), et un décalage de la phase de lecture de l'ARN.

miquement très lourde. À la lumière des progrès des connaissances et des méthodologies, les premiers résultats peu concluants obtenus en systèmes acellulaires en 1993 [1] pourraient être reconsidérés; notamment en essayant d'atteindre d'autres cibles que la mutation elle-même (point de branchement, séquences régulatrices d'épissage). L’hémoglobinose $\varepsilon$ avec son épissage anormal (site donneur anormal créé au codon 26 de l'exon 1) est presque toujours assez bénigne. Cependant l'hétérozygotie composite avec une autre mutation thalassémique entraîne un phénotype sévère qui justifierait son ciblage. D'autres mutations thalassémiques perturbant l'épissage normal sont potentiellement justiciables d'une épissothérapie et méritent l'attention.

\section{Les AON vectorisés}

L'action sur le préARNm des AON administrés tels quels est de courte durée et implique pour les maladies génétiques un apport régulier dont on ignore pour l'instant la fréquence optimale et l'éventuelle toxicité à long terme. C'est pourquoi on a en parallèle tenté d'obtenir une production permanente in situ des AON correcteurs grâce à des constructions camouflées dans une séquence naturelle d'ARN nucléolaire ou snARN, en particulier la séquence U7, et incorporées dans un vecteur viral [21]. Cette stratégie a déjà fait la preuve expérimentale de son efficacité prolongée pour le saut d'exon thérapeutique dans un modèle murin de dystrophinopathie [22, 23-26]. Mais ici, 


\begin{tabular}{|c|c|c|c|c|}
\hline $\begin{array}{l}\text { Localisation } \\
\text { de la mutation }\end{array}$ & Mutation & $\begin{array}{l}\text { Conséquence } \\
\text { moléculaire** }\end{array}$ & Géographie & Remarques \\
\hline \multicolumn{5}{|l|}{ Mutations introniques } \\
\hline \multirow[t]{2}{*}{ IVS1 } & $110 G>A$ & $\begin{array}{c}\text { Création d'un site accepteur } \\
\text { aberrant }\end{array}$ & Bassin méditerranéen & Fréquente et grave \\
\hline & $116 \mathrm{~T}>\mathrm{G}$ & $\begin{array}{c}\text { Création d'un site accepteur } \\
\text { aberrant }\end{array}$ & Bassin méditerranéen & \\
\hline \multirow[t]{4}{*}{ IVS2 } & $654 C>T *$ & Création d'un pseudo-exon & Chine & Fréquente et grave \\
\hline & $705 \mathrm{~T}>\mathrm{G}$ & Création d'un pseudo-exon & Bassin méditerranéen & Sporadique \\
\hline & $745 C>G$ & Création d'un pseudo-exon & Bassin méditerranéen & Assez fréquente \\
\hline & $837 \mathrm{~T}>\mathrm{G}$ & $\begin{array}{c}\text { Création d'un pseudo-exon } \\
\text { (théorique) }\end{array}$ & Inde & \\
\hline \multirow[t]{5}{*}{ Mutations exoniques } & $\begin{array}{l}\text { Codon } 10: \\
\text { GGC }>\text { GCA }\end{array}$ & $\begin{array}{c}\text { Activation d'un site cryptique } \\
\text { (théorique) }\end{array}$ & Inde & \\
\hline & $\begin{array}{l}\text { Codon 19: } \\
A A C>A G C\end{array}$ & $\begin{array}{c}\text { Activation d'un site cryptique } \\
\text { donneur dans le codon } 18\end{array}$ & Malaisie & Hb Malay \\
\hline & $\begin{array}{l}\text { Codon } 24: \\
\text { GGT > GGA }\end{array}$ & $\begin{array}{c}\text { Activation d'un site cryptique } \\
\text { donneur dans le codon } 25\end{array}$ & Afro-Américains & \\
\hline & $\begin{array}{l}\text { Codon 26: } \\
\text { GAG }<A A G\end{array}$ & $\begin{array}{c}\text { Activation d'un site cryptique } \\
\text { donneur dans le codon } 25\end{array}$ & Asie & $\mathrm{Hb} \varepsilon$ \\
\hline & $\begin{array}{l}\text { Codon } 27: \\
\text { GCC > TCC }\end{array}$ & $\begin{array}{c}\text { Activation d'un site cryptique } \\
\text { donneur dans le codon } 25\end{array}$ & Bassin méditerranéen & Hb Knossos \\
\hline
\end{tabular}

Tableau I. Principales mutations du gène HBB génératrices de bêta-thalassémie accessibles à une épissothérapie par masquage du site aberrant. * Mutation traitée dans l'article de Svasti et al. (2009) [12]. ** Expérimentalement prouvée dans tous les cas sauf ceux indiqués en italique. Les meilleurs candidats à l'épissothérapie sont les mutations créant un pseudo-exon au sein d'un intron (fond coloré bleu). Les mutations exoniques peuvent activer un site cryptique donneur résident, soit au codon 18, soit au codon 25. Leur masquage par AON n'a pas encore donné lieu à publication.

la nécessité de recourir à un vecteur viral représente encore un obstacle pour le passage aux essais cliniques.

$\varepsilon$ n attendant, à la lumière des résultats obtenus d'abord dans les dystrophinopathies [8, 24], et maintenant dans certaines formes de thalassémie [12], c'est l'administration systémique d'AON qui porte les espoirs thérapeutiques les plus immédiats. $\diamond$

Update on RNA splicing repair: applications to beta thalassemia and other perspectives

\section{CONFLIT D'INTÉRÊTS}

Les auteurs déclarent n'avoir aucun conflit d'intérêts concernant les données publiées dans cet article.

\section{RéFÉRENCES}

1. Dominski Z, Kole R. Restoration of correct splicing in thalassemic pre-mRNA by antisense oligonucleotides. Proc Natl Acad Sci USA $1993 ; 90$ : 8673-77.

2. Bauman J, Jearawiriyapaisarn N, Kole R. Therapeutic potential of splice-switching oligonucleotides. Oligonucleotides 2009; $19: 1-14$

3. Lebleu B, Moulton HM, Abes R, et al. Cell penetrating peptide conjugates of steric block oligonucleotides. Adv Drug Deliv Rev 2008 ; 60 : 517-29.

4. Aartsma-Rus A, Van Vliet L, Hirschi M, et al. Guidelines for antisense oligonucleotide design and insight into splice-modulating mechanisms. Mol Ther 2009; $17: 548-553$.

5. Tazi J, Bakkour N, Stamm, S. Alternative splicing and disease. Biochim Biophys Acta 2009; 1792 : 14-26.

6. Van Ommen GJ, van Deutekom J, Aartsma-Rus A. The therapeutic potential of antisense-mediated exon skipping. Curr Opin Mol Ther 2008 ; 10 : 140-9.

7. Yokota T, Takeda S, Lu QL, et al. A renaissance for antisense oligonucleotide drugs in neurology: exon skipping breaks new ground. Arch Neurol 2009; $66: 32-8$.
8. Van Deutekom JC, Janson AA, Ginjaar IB, et al. Local dystrophin restoration with antisense oligonucleotide PR0051. N Engl J Med 2007 ; 357 : 2677-86.

9. Kinali M, Arechavala-Gomeza V, Feng L, et al. Local restoration of dystrophin expression with the morpholino oligomer AVI-4658 in Duchenne muscular dystrophy: a single-blind, placebo-controlled, doseescalation, proof-of-concept study. The Lancet Neurology $2009 ; 8$ : 918-28.

10. Forget $B G$. Molecular mechanisms of betathalassemia. In : Steinberg MH, Forget BG, Higgs DR, Nagel RL, eds. Disorders of hemoglobin. Cambridge: Cambridge University Press 2001 : 252-66.

11. Xie SY, Ren ZR, Zhang JZ, et al. Restoration of the balanced alpha/beta-globin gene expression in beta 654-thalassemia mice using combined RNAi and antisense RNA approach. Hum Mol Genet 2007 ; $16: 2616-25$.

12. Svasti S, Suwanmanee T, Fucharoen, S, et al. RNA repair restores hemoglobin expression in IVS2-654 thalassemic mice. Proc Natl Acad Sci USA 2009; $106: 1205-10$.

13. HbVar. http://globin.cse.psu.edu/hbvar/menu.html. 
14. Hayakawa J, Ueda T, Lisowski, et al. Transient in vivo beta-globin production after lentiviral gene transfer to hematopoietic stem cells in the nonhuman primate. Hum Gene Ther $2009 ; 20$ : 563-72.

15. Huang SZ, Zeng Fy, Ren ZR, et al. RNA transcripts of the beta-thalassaemia allele IVS-2-654 C->T: a small amount of normally processed beta-globin mRNA is still produced from the mutant gene. Br J Haematol $1994 ; 88: 541-6$

16. Lacerra G, Sierakowska H, Carestia C, et al. Restoration of hemoglobin A synthesis in erythroid cells from peripheral blood of thalassemic patients. Proc Natl Acad Sci USA 2000 ; 97 : 9591-6.

17. Gong L, Gu XF, Chen YD. Reversal of aberrant splicing of beta-thalassaemia allele (IVS-2-654 C-->T) by antisense RNA expression vector in cultured human erythroid cells. BrJ Haematol 2000 ; 111 : 351-8.
18. Suwanmanee T, Sierakowska H, Lacerra R, et al. Restoration of human beta-globin gene expression in murine and human IVS2-654 thalassemic erythroid cells by free uptake of antisense oligonucleotides. Mol Pharmacol $2002 ; 62$ : 545-53.

19. Vacek MM, Ma H, Gemignani F, et al. High-level expression of hemoglobin in a human thalassemic erythroid progenitor cells following lentiviral vector delivery of an antisense snRNA. Blood 2003 101: 104-11.

20. Lewis J, Yang B, Kim R, et al. A common human beta globin splicing mutation modeled in mice. Blood 1998; 91 : 2152-6.

21. Gorman L, Suter D, Emerick V, et al. Stable alteration of pre-mRNA splicing patterns by modified U7 small nuclear RNAs. Proc Natl Acad Sci USA 1998 ; $95: 4929-34$
22. Goyenvalle A, Vulin A, Fougerousse F, et al. Rescue of dystrophic muscle through U7 snRNA-mediated exon skipping. Science 2004 ; 306 : 1796-9.

23. Benchaouir R, Meregalli M, Farini A, et al. Restoration of human dystrophin following transplantation of exon-skipping-engineered DMD patient stem cells into dystrophic mice. Cell Stem Cell 2007 ; 1: 646-57.

24. Yokota T, Lu QL, Partridge, et al. Efficacy of systemic morpholino exon-skipping in Duchenne dystrophy dogs. Ann Neurol 2009; 65 : 667-76.

25. Kaplan JC, Chelly J, Garcia L. Un saut symbolique mais encourageant dans le traitement de la myopathie de Duchenne. Med Sci (Paris) 2008 ; 24 : 215-7.

26. Goyenvalle A, Vulin A, Fougerousse F, et al. Le saut d'exon thérapeutique: un espoir pour les dystrophinopathies. Med Sci (Paris) 2004 ; $20: 1163-5$.

\section{Collection SCIENCE ET BIOMÉDECINE}

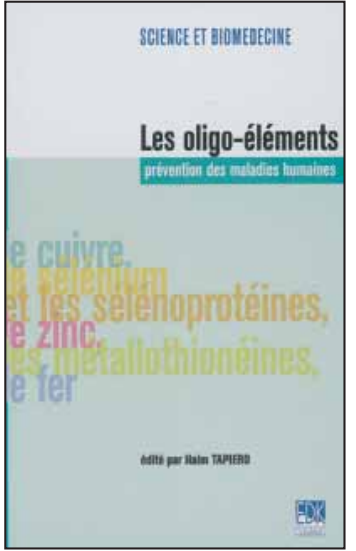

ISBN : 2-84254-107-3 64 pages

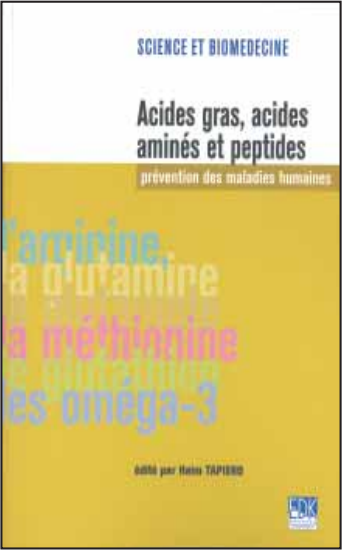

ISBN : 2-84254-108-1 80 pages

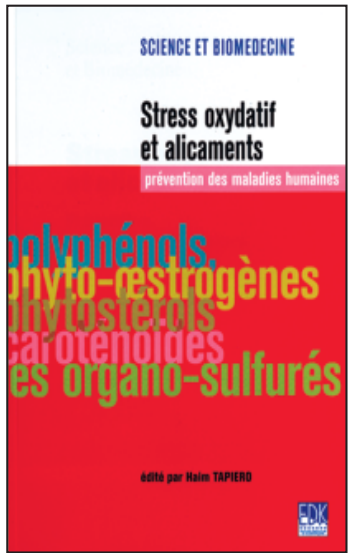

ISBN : 2-84254-111-1 86 pages

\section{Bon de commande}

À retourner à EDK, 2, rue Troyon - 92316 Sèvres Cedex

Tél. : 0155641393 - Fax : 0155641394 - E-mail : edk@edk.fr

NOM :

Prénom :..

Adresse :

Code postal :

Ville :

Pays :

Fonction :

Je souhaite recevoir l'ouvrage Les oligo-éléments : $10 €+3 €$ de port $=\mathbf{1 3} €$ TTC

Je souhaite recevoir l'ouvrage Acides gras, acides aminés et peptides : $12 €+3 €$ de port $=\mathbf{1 5} €$ TTC

Je souhaite recevoir l'ouvrage Stress oxydatif et alicaments : $14 €+3 €$ de port $=\mathbf{1 7} €$ TTC

en ................ exemplaire, soit un total de

Par chèque, à l'ordre de $\mathbf{E} \mathbf{D} \mathbf{K}$

$\square$ Par carte bancaire :

$\square$ Visa $\square$ Eurocard/Mastercard

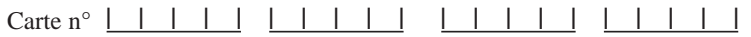
Signature :

Date d'expiration : $\quad 1 \quad 1 \quad 1 \quad 1 \quad$

$\mathrm{N}^{\circ}$ de contrôle au dos de la carte : $\quad|\quad| \quad \mid$ 